\title{
Correction to: Development of Transgenic Cotton for Combating Biotic and Abiotic Stresses
}

Babar Hussain and Sultan Mahmood

\section{Correction to:}

Chapter 26 in: S. Ahmad, M. Hasanuzzaman (eds.),

Cotton Production and Uses, https://doi.org/10.1007/978-981-15-1472-2_26

The original version of the chapter was inadvertently published with incorrect sequence of the authors. The correct sequence is

Babar Hussain and Sultan Mahmood

In addition, Dr. Babar Hussain's email was incorrectly linked with his coauthor. These errors have now been corrected with this erratum. 